\title{
Respuesta de Rivera y colaboradores a la carta al editor de Arroyo y colaboradores sobre consumo de bebidas
}

Señor editor: Agradecemos la oportunidad que nos proporciona la carta al editor de la Fundación Mexicana para la Salud (Arroyo y colaboradores) para responder a las inquietudes y preocupaciones expuestas respecto a nuestro artículo ${ }^{1}$.

La carta al editor de Arroyo y colaboradores incluye un cuerpo principal y dos apartados, por lo que siguiendo el mismo estilo de los responsables de dicha carta, dividiremos nuestra respuesta en dos secciones: la primera, que responderá los puntos tratados en el cuerpo principal de la carta al editor, y una segunda (Respuesta a los dos apartados de la carta de Arroyo y colaboradores), que responderá los puntos contenidos en los dos apartados.

Los autores reconocen al igual que nosotros que las bebidas con aporte calórico constituyen un factor importante en el aumento de peso observado en México. Existe evidencia, presentada en nuestro artículo, ${ }^{1}$ indicando que además de los efectos en el aumento en sobrepeso y obesidad, las bebidas con aporte calórico incrementan el riesgo de diabetes tipo 2. ${ }^{2-6}$ Más aún, después de la publicación de nuestro trabajo se publicó un artículo ${ }^{7}$ mostrando aumento en el riesgo de dia- 
betes tipo 2 en mujeres afroamericanas que consumían regularmente bebidas azucaradas y jugos de frutas. Las concentraciones elevadas de glucosa en sangre y el indice de masa corporal elevado son dos de los principales factores de riesgo de muerte y discapacidad prematuras en los mexicanos; en conjunto estos factores contribuyen con $26.3 \%$ del total de muertes y $10.1 \%$ del total de Años de Vida Ajustados por Discapacidad (AVAD). ${ }^{8}$ Por tanto, el consumo frecuente de bebidas con aporte calórico, que representan la quinta parte de la energía que consumen los mexicanos, constituye un importante factor de riesgo para la salud en México.

Arroyo y colaboradores concuerdan con nosotros respecto a la necesidad de recomendaciones sobre el consumo de bebidas. Como lo indicamos en nuestro artículo, concordamos en que también existen otros factores responsables de la epidemia de obesidad y que los esfuerzos para su prevención y control deben incluir estrategias para incidir en esos otros factores y no solamente en el consumo de bebidas con aporte calórico. Las recomendaciones sobre consumo de bebidas que se publicaron en Salud Pública de México forman parte de una estrategia nacional para la prevención de obesidad que la Secretaría de Salud está implementando gradualmente y que incluye acciones para modificar los principales factores de riesgo de obesidad y enfermedades crónicas.

Varios de los puntos incluidos en la carta al editor fueron planteados directamente a la Secretaria de Salud, a la Comisión Federal para la Protección contra Riesgos Sanitarios (COFEPRIS) y a este comité de expertos por representantes de la industria de alimentos de México. Le damos la bienvenida a la presentación de estos mismos puntos en una carta abierta al editor de Salud Pública de México, lo que nos permite presentar nuestros argumentos y reiterar la validez de la evidencia científica en los que se basan. Este debate académico sin duda contribuirá a fortalecer las recomendaciones y, especialmente, su traducción en políticas públicas de salud para la prevención de obesidad y enfermedades crónicas.
La afirmación de los responsables de la publicación de que las recomendaciones en nuestro artículo son "idénticas" a las publicadas en el American Journal of Clinical Nutrition (AJCN) no solamente es errónea (como lo demostramos más adelante), sino que denota falta de conocimiento sobre la naturaleza y el proceso de desarrollo de recomendaciones con base en evidencia. La tarea que nos encomendó el secretario de Salud fue el desarrollo de recomendaciones sobre bebidas para la población mexicana, considerando evidencia científica sobre riesgos y beneficios para la salud y la nutrición, a la luz de la transición nutricional que nuestro país enfrenta. Para cumplir con dicha encomienda realizamos una revisión exhaustiva de la literatura científica. Como en cualquier ejercicio de revisión de literatura, nuestro punto de partida fue la mejor revisión de literatura sobre el tema de bebidas y salud disponible, la cual era, a nuestro juicio, el artículo del AJCN en el que participaron dos de nuestros coautores. ${ }^{9}$

Dado que la revisión de literatura del AJCN es relativamente reciente, una buena parte de los hallazgos publicados en él son aún vigentes y por tanto se reprodujeron con permiso de la revista, lo cual se hace explícito en la página frontal de nuestro artículo. Vale la pena notar que la biología funciona en forma similar en los humanos, a pesar de las fronteras; de modo que varios de los factores que tienen efectos adversos en la salud de los estadounidenses o los europeos los tienen también en la salud de los mexicanos; no es de extrañar, por tanto, que varias de las conclusiones derivadas del análisis de la literatura científica universal sean similares en ambas publicaciones.

Nos llama la atención que Arroyo y colaboradores consideren que no puede haber coincidencia en las recomendaciones entre dos países, cuando la evidencia universal utilizada para emitir recomendaciones es única.

El segundo paso fue realizar un trabajo minucioso de actualización de la revisión de la literatura, agregando nueva evidencia científica no incluida en la revisión del $\mathrm{AJCN}$, lo que condujo a conclusiones y recomendaciones que difieren de las publicadas en el AJCN, como se ilustra más adelante. Además, nuestro artículo incluyó una sección amplia con información original sobre el consumo de bebidas en México, derivada del análisis de las Encuestas Nacionales de Nutrición de 1988 y 1999 y la Encuesta Nacional de Salud y Nutrición 2006 y de análisis de la serie de Encuestas Nacionales de Ingreso y Gasto en los Hogares. Este análisis específico para la población mexicana permitió el desarrollo de recomendaciones y conclusiones aplicables a la situación epidemiológica de nuestro país.

El proceso que utilizamos, que incluyó la revisión de la literatura, su interpretación y síntesis, el estudio de la situación nacional y la adaptación del conocimiento actual a la situación local, es el proceso recomendado por varios autores en la literatura sobre diseño de políticas de salud con base en evidencia. ${ }^{10-12}$

Debido a que nuestras recomendaciones, así como las publicadas en el artículo de Popkin y colaboradores ${ }^{9}$, se basan en revisiones de la literatura universal disponible sobre los riesgos y beneficios para la salud y nutrición de diversas bebidas, existen similitudes, en la medida en que la evidencia científica disponible no sufrió modificaciones en el período que transcurrió entre ambas publicaciones (la publicación del AJCN fue en el 2006); pero también tienen notables diferencias cuando así se justificó, por haber encontrado nueva evidencia científica, no disponible en la publicación del AJCN, o debido a diferencias entre la población mexicana y la estadounidense en cuanto a patrones de consumo de bebidas o en la situación nutricional y de salud. Varias de las diferencias se detallan más adelante; incluimos aquí algunos ejemplos.

a) Nuestra revisión de literatura incluyó dos metaanálisis, no incluidos en el AJCN, con evidencia clara sobre aumento en el riesgo de obesidad y diabetes tipo 2 asociado al consumo de bebidas con alto contenido de azúcares ${ }^{6,13}$. Esto llevó a la siguiente 
recomendación respecto a las bebidas con azúcar y bajo contenido de nutrimentos (nivel 6): “...no se recomienda su consumo; si se ingieren debe ser sólo de manera ocasional ..." En cambio, la publicación del $\mathrm{AJCN}^{9}$ recomienda el consumo de hasta 8 onzas por día de estas bebidas.

b) Debido a la alta prevalencia de sobrepeso en los menores de 5 años y a su aumento en las dos últimas décadas, especialmente en los grupos de mayores ingresos y en zonas urbanas, nuestro artículo presenta recomendaciones específicas para niños de 2 a 5 años, diferentes de las recomendaciones para adultos (ver la descripción en detalle en la primera sección de Respuesta a los apartados de Arroyo y colaboradores). La publicación del $\mathrm{AJCN}^{9}$, en cambio, no presenta recomendaciones para dicho grupo de edad.

c) Debido a la existencia de la doble carga de la desnutrición y la mala nutrición por exceso en México, se hacen recomendaciones diferenciales sobre consumo de leche entera en niños de 2 a 4 años para zonas con altas o con bajas prevalencias de desnutrición. La publicación del $\mathrm{AJCN}^{9}$ no hace dicha distinción.

d) Dado que algunos de los edulcorantes artificiales fueron introducidos apenas en la década de los ochenta, hay evidencia limitada acerca de sus efectos a largo plazo, particularmente en niños. Además, existe evidencia de que los sabores dulces condicionan las preferencias por alimentos y bebidas dulces posteriormente en la vida. Por tanto, recomendamos que los niños no consuman estas bebidas y que los adultos no consuman más de $500 \mathrm{ml}$ por día, a diferencia de la publicación del AJCN que recomienda hasta 32 onzas (alrededor de 1000 $\mathrm{ml}$ ) por día para adultos y no emite recomendaciones específicas para niños.

e) Debido a nueva evidencia disponible sobre el aumento en riesgo de obesidad y diabetes tipo 2 asociada al consumo de jugos de frutas y al alto consumo de dichas bebidas en México, recomendamos en nuestra publicación el consumo de no más de $125 \mathrm{ml}$ de jugos de fruta al día y enfatizamos el consumo de frutas enteras en lugar de licuarlas. En cambio la publicación del $\mathrm{AJCN}^{9}$ recomienda hasta 8 onzas (alrededor de $240 \mathrm{ml}$ ) de jugos de fruta por día.

f) Nuestro artículo incluye una serie de directrices sugeridas a la Secretaría de Salud para ser consideradas y discutidas con los sectores involucrados, con el propósito de definir su viabilidad y los pasos necesarios para su aplicación (págs. 191 y 192 de nuestro artículo), las cuales no se incluyen en la publicación del $\mathrm{AJCN}^{9}$.

Dado que hemos demostrado que las recomendaciones en nuestro artículo no son idénticas a las publicadas en el AJCN, como señalan equivocadamente los responsables de la carta al editor, el supuesto de que los problemas atribuidos a la publicación del AJCN son también atribuibles a nuestro trabajo es también erróneo. Este supuesto equivocado originó varios comentarios errados, muchos de ellos transcritos verbatim de comentarios hechos por otros autores al artículo del AJCN, sin considerar el contenido de nuestro trabajo publicado en Salud Pública de México, y que por tanto no aplican al mismo, como mostramos detalladamente en Respuesta a los apartados de Arroyo y colaboradores.

Un hecho importante es que los responsables de la carta al editor presentan la postura de un sector de la industria de alimentos, la cual difiere de algunas de nuestras recomendaciones, pero no hacen alusión y no disputan la evidencia científica que avala dichas recomendaciones. La mayor parte de las críticas de los autores en relación a nuestras recomendaciones son opiniones que no consideran la evidencia publicada en la literatura científica sobre los efectos de diversas categorías de bebidas en la salud y la nutrición. El debate académico sería más útil si al presentar el punto de vista de un sector de la industria de alimentos, los autores de la carta hubieran disputado la evidencia científica que avala nuestras recomendaciones, en lugar de emitir opiniones sin respaldo científico.

Los responsables de la carta al editor manifiestan inquietud sobre nuestra decisión de incluir recomendaciones para niños de 2 a 5 años de edad. Nuestra decisión se basó en la alta prevalencia actual de sobrepeso y obesidad en este grupo de edad, en su aumento en las dos últimas décadas y en el alto consumo de bebidas con aporte calórico en este grupo de edad. Sin embargo, contrario a lo que afirman erróneamente Arroyo y colaboradores, las recomendaciones para niños no son las mismas que las recomendaciones para adultos. Para la mayor parte de las categorías de bebidas incluimos secciones específicas e hicimos recomendaciones especiales dirigidas a este grupo de edad (ver varios ejemplos en la primera sección de Respuesta a los apartados de Arroyo y colaboradores). Más aún, las recomendaciones ilustradas en las figuras 5 y 6 de nuestra publicación se refieren solamente a la población adulta, como se especifica claramente en sus títulos y en el texto, lo que los responsables de la carta parecen no haber advertido. Por tanto, nuestras recomendaciones hacen precisamente lo que los responsables de la carta al editor sugieren que deberíamos haber hecho: diseñar recomendaciones específicas para niños, las cuales son diferentes a las recomendaciones para adultos, cuando así se justifica. Además, estamos en desacuerdo con los responsables de la carta en el sentido de que las recomendaciones de los adultos aplican a niños entre 6 y 18 años; algunas recomendaciones (por ejemplo, consumo de alcohol o café) difieren para estos grupos de edad. En Respuesta a los apartados de Arroyo y colaboradores presentamos las recomendaciones específicas para niños, publicadas en nuestro artículo, con el propósito de probar nuestro argumento.

Los responsables de la carta opinan erróneamente que las "recomendaciones idénticas para Estados Unidos y México" 
pueden deberse a que solamente uno de los miembros del comité es nutriólogo. Discrepamos de la visión de Arroyo y colaboradores de que solamente individuos entrenados como nutriólogos o en ciencias sociales tienen la capacidad para participar en el diseño de recomendaciones, basadas en evidencia, sobre consumo de bebidas. La necesidad de conocer a fondo la literatura y entender la relación entre dieta, estado nutricio y enfermedades crónicas es fundamental en el desarrollo de recomendaciones con base en evidencia sobre el consumo de bebidas, diseñadas con el propósito de reducir el riesgo de obesidad y enfermedades crónicas. Las publicaciones de los seis miembros del grupo de expertos hacen patente una amplia experiencia en investigación científica sobre la relación entre la nutrición y las enfermedades crónicas y en el diseño y evaluación de políticas en salud.

El grupo convocado por la Secretaría de Salud, en una decisión clara de basar las decisiones en la mejor evidencia científica, incluye tanto a expertos nacionales como internacionales. Entre sus elementos está uno de los autores más importantes de la literatura sobre dieta y su relación con la salud y la enfermedad (W. Willett), quien dirige varios de los estudios de cohorte más importantes sobre dieta y enfermedad, que han servido de base para el desarrollo de recomendaciones internacionales sobre dieta, como las del World Cancer Research Fund (WCRF) ${ }^{13}$ y varios reportes de la Organización Mundial de la Salud (OMS). El grupo también incluyó a un economista de alimentos (B. Popkin), con entrenamiento y experiencia en análisis demográfico, quien desarrolló el concepto de "transición nutricional" y ha estudiado este fenómeno en varios países, incluyendo México. Tanto J Rivera como W. Willett formaron parte del Comité de Expertos a cargo del desarrollo del libro "Food, nutrition, physical activity, and the prevention of cancer: a global perspective" del WCRF, el cual incluyó un metaanálisis sobre los factores de riesgo de obesidad ${ }^{13} \mathrm{y}$ ambos investigadores, así como B. Popkin, forman parte del grupo de expertos a cargo del desarrollo de la publicación sobre políticas de alimentación y actividad física del mismo WCRF. Además, formaron parte del grupo dos científicos con vasta experiencia en investigación clínica y epidemiológica y con varias publicaciones sobre los determinantes de dos de las enfermedades responsables de una importante proporción de la carga de enfermedad en México: diabetes (C. Aguilar-Salinas) y enfermedad cardiovascular (M. Rosas). Estos investigadores se desempeñan en dos de los Institutos Nacionales de Salud con mayor prestigio y experiencia en la investigación sobre estas dos enfermedades crónicas: el Instituto Nacional de Ciencias Médicas y Nutrición Salvador Zubirán y el Instituto Nacional de Cardiología Ignacio Chávez. Finalmente, el grupo incluye a un pediatra, experto en investigación en sistemas de salud, con amplio conocimiento sobre políticas públicas de salud en México, que actualmente se desempeña en otro de los Institutos Nacionales de Salud, el Hospital Infantil de México Federico Gómez (O. Muñoz). A diferencia de la visión de los responsables de la carta al editor, en el sentido de que sólo los nutriólogos están calificados para realizar recomendaciones con base en videncia sobre bebidas, considerando sus efectos en la nutrición y la salud y enfermedad, la trayectoria de los participantes indica que fue adecuada la decisión de la Secretaría de Salud de seleccionar para el desarrollo de las recomendaciones a este grupo multidisciplinario, conocedor de la literatura sobre alimentación, nutrición y salud.

Siguiendo el modelo de recomendaciones internacionales prestigiadas, como las del WCRF, otros actores relevantes participarán en el ejercicio de la traducción de nuestras recomendaciones hacia políticas y acciones de salud, como parte del Plan Nacional de Prevención de Obesidad y Enfermedades Crónicas que la Secretaría de Salud está implementando gradualmente. En nuestra publicación establecemos esto claramente al afirmar que "se presentan algunas directrices sugeridas por el Comitéa la Secretaría de Salud para ser consideradas y discutidas con los sectores involucrados, con el propósito de definir su viabilidad y los pasos necesarios para su aplicación". ${ }^{1}$

Es claro que nuestro artículo proporciona recomendaciones basadas en la mejor evidencia científica disponible y que son válidas para la población mexicana. Aunque éstas podrían afectar intereses económicos, consideramos que nuestra responsabilidad, así como la de otros investigadores en el campo de la salud y la Secretaría de Salud, debe ser la de velar por la salud de los mexicanos y reforzar la prevención de la obesidad y la promoción de patrones de alimentación saludable y de actividad física. Reconocemos la importancia que tiene la industria alimentaria en lo que corresponde a la prevención de la obesidad. Por esa razón resulta imprescindible su participación decidida para fortalecer los esfuerzos de la Secretaria de Salud, dirigidos a la prevención y control de la obesidad, la diabetes y otras enfermedades crónicas, así como su apoyo a las recomendaciones basadas en evidencia.

Consideramos que la industria puede contribuir al combate a la obesidad y las enfermedades crónicas de varias maneras; por ejemplo, mediante la reducción gradual y concertada del contenido de azúcar y de grasas saturadas en las bebidas que producen; mediante la restricción de la disponibilidad de bebidas con alto contenido de energía en las escuelas; y evitando estrategias de mercadeo dirigidas a promover estos productos entre los niños. Finalmente, hacemos un llamado a la comunidad interesada en la salud pública, a la industria y a la sociedad civil a unir esfuerzos en la prevención y control de la obesidad y las enfermedades crónicas. Ante la grave epidemia de obesidad y diabetes que aqueja a nuestro país, y sus graves consecuencias sociales, hoy más que nunca debemos trabajar juntos en la prevención de estos padecimientos.

\section{RESPUESTA A LOS DOS APARTADOS DE} LA CARTA DE ARRoyo y COLABORADORES

Antes de dar respuesta puntual a los comentarios de los responsables de la carta al editor en sus apartados Popkin y colaboradores y Rivera y colaboradores, presentamos las recomendaciones específicas 
para niños que, al parecer, no advirtieron los responsables de la carta.

\section{Recomendaciones para niños de 2-5 años}

Los responsables de la carta al editor afirman que no diseñamos recomendaciones específicas para niños de 2 a 5 años. Esta afirmación es incorrecta. A continuación presentamos recomendaciones específicas publicadas en nuestro artículo. Como se mencionó antes, las recomendaciones ilustradas en las figuras 5 y 6 de nuestra publicación ${ }^{1}$ aplican sólo para adultos, como se indica claramente en sus títulos y en el texto. Aportamos aquí ejemplos de recomendaciones específicas para niños, seguidas de la página en nuestro artículo en el que pueden encontrarse.

Café y té. Presentamos la recomendación de no más de $2.5 \mathrm{mg} / \mathrm{kg} /$ día de cafeína para niños. Estas son cantidades muy pequeñas. En la práctica, significa que los niños de 2-5 años no deben consumir café o té (p. 183).

Bebidas endulzadas sin aporte calórico. Dado que algunos de los edulcorantes artificiales fueron introducidos apenas en la década de los ochenta, hay evidencia mínima acerca de sus efectos a largo plazo, particularmente para niños. Además, hay evidencia de que los sabores dulces condicionan las preferencias por los alimentos y bebidas dulces posteriormente en la vida. Por tanto, recomendamos que los niños no consuman bebidas con edulcorantes artificiales (p. 184).

Leche entera. Aunque recomendamos el consumo de leche baja en grasa o sin grasa para los individuos de 2 años o más, la excepción, mencionada claramente, son los niños que viven en áreas con altas prevalencias de desnutrición; en estos niños se recomienda el consumo de leche entera (p. 185)

Bebidas alcohólicas. Las recomendaciones son sólo para adultos (p. 185).

\section{Respuesta a los comentarios en el apartado Popkin y colaboradores}

En el apartado Popkin y colaboradores, los responsables de la carta al editor toman literalmente críticas hechas al artículo del AJCN, las cuales fueron respondidas por los autores en su momento. Desafortunadamente, salvo algunas excepciones, los responsables de la carta no indican cuales de esas críticas aplican a nuestro artículo. En lugar de referirnos al artículo del AJCN y las críticas que de éste se hicieron, nos concentraremos exclusivamente en las críticas que a nuestro juicio aplican a nuestra publicación.

Una de ellas es el desacuerdo de los responsables de la carta sobre el concepto de densidad energética de las bebidas. Hemos definido densidad energética como el contenido de energía por unidad de volumen. Los conceptos publicados en nuestro artículo en relación con el contenido energético de las bebidas coinciden completamente con las de Rolls (citada en la carta el editor, erróneamente, como con una postura diferente a la nuestra), quien ha realizado estudios que incluimos en nuestra revisión. Sus conclusiones sobre los efectos de las bebidas sobre obesidad coinciden con las nuestras. ${ }^{14-15}$ Presentamos evidencia en nuestro artículo (p. 175) de que los líquidos con alto contenido energético tienen menor capacidad para producir saciedad y compensación dietética que los alimentos sólidos con el mismo contenido energético; ${ }^{16}$ por tanto, nuestra preocupación sobre el contenido energético de las bebidas (densidad energética) se fundamenta en sus efectos sobre el consumo total de energía, dada su baja capacidad de inducir compensación dietética. Más aún, es claro que las bebidas con alta densidad de energía (particularmente las clasificadas en nuestro artículo como de nivel 6) aumentan el riesgo de obesidad y diabetes tipo 2 y provocan otros efectos adversos en la salud. . $^{2-5,17,18}$ Cabe resaltar dos metaanálisis recientes, ${ }^{6,}$ ${ }^{13}$, posteriores a la publicación del artículo del AJCN, los cuales muestran claros efectos del consumo de bebidas con aporte calórico en aumentos en el riesgo de sobrepeso, obesidad y diabetes tipo 2 . Nuestra decisión de utilizar el contenido de energía por unidad de volumen, como uno de los criterios (entre otros) para clasificar a las bebidas en los diferentes niveles presentados en nuestro artículo, está sustentada en la evidencia sobre los efectos adversos de las bebidas con aporte calórico en la salud. Entre mayor contenido de energía por unidad de volumen (mayor densidad) mayor es el riesgo de sobrepeso y obesidad. Los responsables de la carta no indican cuál es el corolario de su desacuerdo con el uso del contenido energético por unidad de volumen en los líquidos, como uno de los criterios para definir su nivel de riesgo en la salud: ¿objetan nuestra recomendación en el sentido de reducir el consumo de las bebidas con alto contenido energético? De acuerdo a lo expresado por los responsables de la carta al editor, coinciden con nosotros en que "las bebidas con valor energético son un factor importante en el aumento del peso corporal". Desconocemos, por tanto, la causa por la que aceptando este postulado estén en desacuerdo de utilizar la densidad energética (valor energético por unidad de volumen) como uno de los criterios para clasificar las bebidas de acuerdo con niveles de riego para el aumento del peso corporal.

En lugar de analizar nuestro artículo y sus fundamentos, los responsables de la carta al editor reproducen críticas realizadas al artículo publicado en el AJCN, la mayor parte de las cuales no aplican a nuestro artículo. Ponemos varios ejemplos: 1) nuestra publicación recomienda el consumo de hasta $500 \mathrm{ml}$ de leche baja en grasa o sin grasa y no considera al café o al té superiores a la leche baja en grasa, como sugieren los responsables de la carta; 2) claramente indicamos que no utilizamos como único criterio para la clasificación de las bebidas su densidad energética, sino que también usamos la densidad nutrimental y evidencia sobre efectos adversos en la salud; 3) identificamos fuentes de calcio diferentes a la leche, como la tortilla, y no recomendamos que la población mexicana dependa exclusivamente de verduras y frutas para satisfacer sus necesidades de calcio. Por el contrario, recomendamos el consumo de hasta $500 \mathrm{ml}$ de leche baja en grasa.

Finalmente, el intercambio entre Kaplan y Popkin sobre conflicto de intereses 
en cartas publicadas en el AJCNy la opinión vertida por los autores de la carta al editor sobre los posibles conflictos de interés del editor de AJCN no son relevante para nuestra publicación, nuestras recomendaciones y la salud pública de nuestro país.

Respuestas a los comentarios en el apartado Rivera y colaboradores

A diferencia de los comentarios del apartado Popkin y colaboradores, los del apartado Rivera y colaboradores son relevantes para nuestro artículo. A continuación presentamos nuestras respuestas a los comentarios.

Nuestra guía sobre el consumo de bebidas tiene similitudes y diferencias con la propuesta por Popkin y colaboradores en el artículo del AJCN, de la misma manera que otros sistemas de guías de alimentos adoptadas por diversos países, tienen similitudes y diferencias. Por ejemplo, varios países usan pirámides de alimentos como una manera de ilustrar sus recomendaciones y adaptan el concepto de pirámide a sus necesidades. El uso de las pirámides de alimentos fue reconocido como un buen instrumento para la guía en varios países, se generalizó su uso y se convirtió en un bien público sin que nadie fuera acusado por haber adoptado la idea. Nuestro grupo encontró suficientes méritos en el sistema propuesto por Popkin y colaboradores e hizo los ajustes considerados como necesarios. Cabe aclarar que el instrumento final de diseminación (parte de la etapa de traducción a políticas) está en proceso de elaboración por parte de la Secretaría de Salud, utilizando tecnología de punta, como investigación formativa y mercadeo social y considerando opiniones de diversos especialistas y las aportadas por la industria de alimentos ante COFEPRIS, reproducidas por Arroyo y colaboradores en su carta.

Acontinuación respondemos afirmaciones y comentarios de los responsables de la carta al editor, los cuales dividen en el apartado Rivera y colaboradores en 9 puntos.

Punto 1. a) Los autores afirman que "el comité no presenta información que refleje una investigación propia sobre el valor nutricio de las bebidas en México". Esta afirmación es falsa. En la publicación presentamos información sobre el contenido de energía y valor nutrimental de las bebidas mexicanas, obtenida de diferentes fuentes en México. Recomendamos a los responsables de la carta al editor revisar el cuadro III de nuestro artículo. Las fuentes incluyen: a) la base de datos de valor nutrimental de alimentos del Instituto Nacional de Salud Pública, b) el contenido de lípidos en leche y productos lácteos publicados por el laboratorio del Instituto Nacional de Salud Pública, c) información a partir de etiquetas de alimentos de productores mexicanos y d) el sistema mexicano de equivalencias. Solamente 8 de 58 bebidas provienen del artículo de Popkin y colaboradores. ${ }^{9}$ El el mismo cuadro III damos crédito a todas las fuentes, incluido el artículo de Popkin y colaboradores, a diferencia de la afirmación de los responsables de la carta al editor. Como cualquier otro grupo encargado de emitir recomendaciones, iniciamos con una revisión de literatura. De acuerdo con investigadores que han publicado en relación con el diseño de políticas con base en evidencia, no deben hacerse recomendaciones con base exclusiva en las investigaciones de los autores de las recomendaciones o con base en investigación conducida únicamente en el país para el cual se desarrollarán las recomendaciones ${ }^{10-12}$; es necesario hacer revisiones de la literatura universal disponible, interpretarla, sintetizarla y adaptarla a la situación local; esta fue precisamente la secuencia que seguimos en nuestra publicación.

b) Estamos de acuerdo en que incluir a la leche entera en las misma categoría de las bebidas alcohólicas es controversial. Este mismo argumento fue expresado por la industria lechera en reuniones con COFEPRIS en las que fuimos invitados a participar. La razón por la que colocamos al alcohol y la leche entera en la misma categoría es porque ambas tienen beneficios para la salud cuando se consumen en cantidades muy moderadas y tienen efectos adversos cuando se consumen en cantidades elevadas. Estamos de acuerdo que en el proceso de traducir las recomendaciones del comité de expertos a una estrategias de diseminación debe tomarse en consideración la sugerencia de separar la leche del alcohol, sin menoscabo de nuestra recomendación de preferir el consumo de leche baja en grasa o sin grasa sobre la leche entera, la cual debe consumirse, si es el caso, en cantidades moderadas. Es necesario recordar que el alcohol está clasificado muy por debajo, en prioridad de consumo, de la leche baja en grasa y la leche sin grasa (las cuales son la segunda opción más saludable después del agua); la razón por la que colocamos a leche entera en una categoría de baja prioridad en el consumo es porque las grasas saturadas aportan cantidades importantes de energía y tienen efectos adversos en la salud (enfermedades cardiovasculares). En casi todos los países desarrollados se recomienda que el consumo de grasas saturadas (presentes en la leche entera) deben reducirse para disminuir el riesgo de enfermedades cardíacas.

c) Los responsables de la carta al editor juzgan incorrecto que se considere a las bebidas con frutas frescas, condenadas al nivel 6, como menos saludables que las bebidas alcohólicas. Como se indica con toda claridad en nuestro artículo, las "bebidas a base de frutas" del nivel 6 son las "aguas frescas", las que contienen en promedio $100 \mathrm{~g}$ de azúcar por litro y cantidades mínimas de jugo de fruta. Por tanto, en términos de su riesgo de obesidad y diabetes se encuentran en el mismo nivel de riesgo que otras bebidas altas en 
azúcar, como los refrescos. Como se mencionó antes, existe evidencia de que las bebidas que contienen cantidades altas de azúcar aumentan el riesgo de obesidad y diabetes. También mencionamos en el artículo que las aguas frescas con bajo contenido de azúcar (por ejemplo 20 gr/ litro) son aceptables. Los jugos de frutas frescas, en cambio, se ubican en el nivel 5 y la recomendación del comité es que su consumo sea moderado (125 ml/día), dado que estudios en varios países indican que el consumo elevado de jugos de frutas aumenta el riesgo de obesidad y diabetes. ${ }^{7,19-21}$

d) Los responsables de la carta al editor consideran debatible que el café y el té (sin azúcar) se sitúen en una categoría de mayor prioridad que los jugos de frutas. Hemos presentado evidencia en el artículo señalando que el café y el té sin azúcar, consumidos dentro de los límites de las cantidades recomendadas (hasta $400 \mathrm{mg}$ de cafeína al día), no aumentan el riesgo de enfermedades crónicas, a diferencia de las bebidas con alto contenido de azúcares, incluyendo los jugos de fruta. Más aún, presentamos evidencia de que el consumo moderado de café se asocia con la prevención de diabetes tipo 2 (p. 183 y 184).

En resumen, todas nuestras recomendaciones se basan en evidencia científica descrita y citada en el artículo. No encontramos en los comentarios de la carta al editor ninguna objeción sobre la calidad de la evidencia usada como base para las recomendaciones. Las objeciones que se presentan en la carta son opiniones ("creemos", "nos parece", "es debatible") que no se basan en evidencia científica. Pueden, sin embargo, ser útiles, al momento de pasar a la fase de la traducción de nuestras recomendaciones a políticas de salud.

Punto 2. Se objeta la edad mínima de la aplicación de recomendaciones.

Como se mencionó antes, nuestra publicación incluye recomendaciones para niños entre 2 y 5 años, dadas las elevadas prevalencias de sobrepeso y obesidad, su aumento en las últimas dos décadas y el aumento en el consumo de bebidas con aporte energético en este grupo de edad.

Estamos de acuerdo con la observación de los responsables de la carta al editor de que la leche es un alimento muy importante para los niños. Nuestro grupo ha demostrado que la leche fortificada con hierro, zinc y otros micronutrimen$\operatorname{tos}^{22} \mathrm{y}$ una papilla fortificada que contiene leche, ${ }^{23}$ administrados a menores de dos años, disminuye la anemia y mejora el estado nutricio.

Sin embargo, a partir de los dos años de edad es conveniente disminuir el consumo de grasas saturadas. Presentamos evidencia en el artículo, proveniente de ensayos aleatorizados y controlados, que muestra que el consumo de leche baja en grasa durante la niñez temprana disminuye el riesgo de enfermedades crónicas. Un grupo de niños alimentados con leche baja en grasa desde los 7 meses hasta los 5 años de edad mostró reducción en las concentraciones de colesterol sérico a los 5 años de edad, comparado con un grupo que recibió leche entera durante el mismo período. A los 7 años el grupo de niños que recibió leche baja en grasa tuvo menor concentración de colesterol total y lipoproteína de baja densidad (LDL por sus siglas en inglés) y a los 9 años el consumo de leche baja en grasa se asoció con un efecto positivo en un índice de resistencia a la insulina. ${ }^{24,25}$ Más aún, la Academia Americana de Pediatría y la Academia Americana del Corazón apoyan la recomendación de proporcionar leche baja en grasa a los niños a partir de los 2 años de edad. ${ }^{26}$

Es verdad que de 1999 a 2006 el grupo de niños de 2-4 años en el ámbito nacional no aumentó su prevalencia de sobrepeso. Esto probablemente se debió al aumento de la talla en los niños mexicanos durante dicho periodo. Sin embargo, la prevalencia nacional de sobrepeso y obesidad en 2006 fue sumamente alta (15.8\%) y aumentó en las últimas dos décadas (de 1988 a 2006) en 6.8\% usando el sistema de clasificación del International Obesity Taskforce (IOTF).* Más aún, el aumento en sobrepeso en los últimos 20 años, usando las nuevas normas de la OMS, publicadas en 2006, es de 23\%. ${ }^{\ddagger}$ Estas altas prevalencias y sus aumentos en una etapa tan temprana de la vida, usando dos diferentes sistemas de clasificación, para un padecimiento que se acumula a lo largo de la vida, son suficiente razón para emprender acciones dirigidas a la prevención de sobrepeso y obesidad. Una observación importante para nuestras recomendaciones fue que las tendencias de los cambios en prevalencias difieren por nivel socioeconómico. Las prevalencias de sobrepeso y obesidad aumentaron en el tercil medio de nivel socioeconómico en $13 \%$ y en el tercil superior en $15 \%$, mientras que en el tercil inferior (el que registró los mayores aumento de talla) se redujeron en $13 \%$. Las prevalencias en los terciles medio y alto de nivel socioeconómico fueron 15.1 y $18.3 \%$, respectivamente, en $2006 .{ }^{*}$

Otro hallazgo, publicado recientemente en Lancet por el Grupo de Estudio sobre Desnutrición Materna e Infantil ${ }^{27}$ es la observación, a partir del análisis de varios estudios de cohorte en países de ingresos bajos y medios, de que los niños con crecimiento restringido durante la gestación y los primeros dos años de vida y que posteriormente aumentan rápidamente de peso tienen mayor probabilidad de tener presión arterial alta, diabetes y enfermedades metabólicas y cardiovasculares en la edad adulta. Es decir, en países como México deben implementarse acciones dirigidas a la prevención del aumento de peso a partir de los dos años de edad.

Por tanto, es claro que se deben iniciar medidas para evitar el incremento de peso después de los dos años, especial-

\footnotetext{
* Bonvecchio A, Safdie M, Monterrubio E, Gust T, Villalpando S, Rivera JA. Overweight and obesity trends in Mexican children 2 to 18 years of age from 1988 to 2006. En prensa.

‡ González de Cossío T, Rivera J, González D, Unar M, Monterrubio E. Child Malnutrition in Mexico in the last 2 decades: prevalences using the new WHO growth standards. En prensa.
} 
mente en la población con crecimiento restringido durante la gestación y los primeros años de vida y para reducir el sobrepeso y la obesidad en los niños en general, especialmente en los dos terciles superiores de nivel socioeconómico, los que han registrado aumentos en prevalencias de sobrepeso y obesidad. * Como lo hicieron notar acertadamente los responsables de la carta al editor, el aumento en el consumo de bebidas con aporte calórico entre 1988 y 2006 en este grupo de edad es principalmente debido al consumo de leche entera. Considerando la doble carga de la desnutrición y la mala nutrición por exceso, nuestra publicación recomienda el consumo de leche entera en los menores de dos años y de leche baja en grasa en los mayores de 2 años, excepto en los niños que viven en zonas en la que la desnutrición sigue siendo un problema de salud pública, en donde la leche entera sigue recomendándose hasta los 4 años de edad. Dada la contundente evidencia aquí presentada, no encontramos justificación alguna para modificar nuestra recomendación sobre consumo de leche entera en niños de 2 a 4 años.

Punto 3. No hay duda de que la leche es una fuente excelente de calcio y por esa razón recomendamos el consumo de 500 $\mathrm{ml}$ por día de leche baja en grasa. Sin embargo, mencionamos en nuestro artículo otras fuentes de calcio en la dieta, además de la leche. La tortilla es una fuente rica en calcio $(175 \mathrm{mg} / 100 \mathrm{~g})$. Según datos de la Encuesta Nacional de Nutrición de 1999, el maíz y sus derivados son la primera fuente de calcio en la dieta de mujeres, aportando alrededor del $36 \%$ del total de dicho mineral (254 g), seguida de la leche, que aporta alrededor del $33 \%$ y los frijoles el $4 \%$. Hay varios vegetales consumidos en la dieta del mexicano que son altos en calcio, incluyendo nopal (190 g/100g), epazote $(275 \mathrm{mg} / 100 \mathrm{~g})$, papaloquelites (360 mg/100g), chipilín (287 mg/100g),

\footnotetext{
* Bonvecchio A, Safdie M, Monterrubio E, Gust T, Villalpando S, Rivera JA. Overweight and obesity trends in Mexican children 2 to 18 years of age from 1988 to 2006. En prensa.
}

hoja santa (257 mg/100g), berros (211 g $/ 100 \mathrm{~g}$ ) y otros productos utilizados para la elaboración de moles y salsas, como la semilla de calabaza $(975 \mathrm{mg} / 100 \mathrm{~g})$. De ninguna manera se recomienda que la población mexicana dependa exclusivamente de estas verduras como fuente única de calcio.

Punto 4. En Australia, país con el consumo más alto de jugos de fruta en niños en el mundo, estos jugos se han vinculado con obesidad. El aumento en la tasa de obesidad de los niños australianos es posiblemente la más alta del orbe ${ }^{19,20} \mathrm{y}$ deseamos evitar que México alcance prevalencias similares. Como se mencionó antes, el consumo de cantidades altas de jugos de frutas se asocia con obesidad y diabetes tipo 2. Debemos fomentar el consumo de frutas en su forma sólida, no el consumo de jugos de frutas.

Punto 5. Con base en la evidencia estudiada, hemos concluido que la mayor parte de las necesidades de fluidos deben satisfacerse con agua. El hecho que muchos mexicanos no tengan acceso a agua potable debe llevar a políticas y programas para garantizar la disponibilidad de agua potable para todos los mexicanos, en lugar de llevar a la recomendación del consumo de bebidas con azúcar. En la fase de traducción de nuestras recomendaciones a políticas, mencionada antes, la Secretaría de Salud está trabajando con la Comisión Nacional del Agua (CONAGUA) para asegurar que la provisión de agua potable se convierta en un derecho universal.

Punto 6. No encontramos en la literatura evidencia de que el consumo de edulcorantes artificiales aumente el riesgo de enfermedad o muerte. Por otro lado, no contamos con información suficiente sobre los efectos a largo plazo de la exposición desde la edad temprana a estos productos. Por tanto, basados en la evidencia, las bebidas con edulcorantes artificiales se asocian con menor riesgo de obesidad y enfermedades crónicas que la leche entera (la cual aumenta el riesgo de obesidad y enfermedades cardiovasculares) y que las aguas frescas con alto contenido de azúcar (las cuales aumentan el riesgo de obesidad y diabetes tipo 2).

Estamos de acuerdo con los responsables de la carta al editor que los niños requieren recomendaciones diferentes a las de los adultos. Como se mencionó anteriormente, las recomendaciones de las figuras 5 y 6 son exclusivamente para adultos, como se indica claramente en el artículo. ${ }^{1}$ Hemos mostrado antes que tenemos recomendaciones diferentes para niños. Por ejemplo, recomendamos que los niños no consuman bebidas con edulcorantes artificiales.

Punto 7. Como se mencionó antes, nuestra publicación recomienda el consumo de hasta $500 \mathrm{ml}$ de leche por día. Sin embargo, los productos lácteos pueden ser substituidos por otros alimentos. Además, los micronutrimentos presentes en la leche pueden ser reemplazados con suplementos farmacológicos. Esto se menciona como posibilidad para quien opte por no consumir leche; aunque nuestra recomendación favorece el consumo de leche baja en grasa. En México varios programas distribuyen actualmente suplementos farmacológicos con micronutrimentos a mujeres embarazadas y a niños, lo que indica la factibilidad de que mexicanos, aun de bajos ingresos, obtengan algunos nutrimentos a partir de suplementos farmacológicos. Reiteramos que nuestra recomendación para satisfacer las necesidades de calcio es hacerlo con una dieta alta en leche baja en grasa y en otros alimentos fuentes de calcio.

Los responsables de la carta al editor cuestionan nuestra afirmación de que "el agua potable carece casi por completo de efectos adversos en los individuos sanos cuando se consume en los intervalos de valores aceptados". Su objeción se basa en que el agua potable "dura" de Yucatán se ha relacionado con endurecimiento de las arterias y litiasis. Insistimos en mantener nuestra afirmación sobre la ausencia casi por completo de efectos adversos del consumo de agua. Los efectos deletéreos del agua sobre la salud son la excepción. En lugares donde el agua contiene altas 
cantidades de minerales o en lugares en los que está contaminada o tiene exceso de flúor, el gobierno debe asegurar acceso a agua adecuada para consumo humano. Existe la tecnología para lograrlo. Los efectos adversos sobre la salud de agua inadecuada para consumo humano no justifican el cambio de nuestras recomendaciones.

Punto 8. Los límites superior e inferior presentados se refieren a aumentos en el consumo de energía proveniente de bebidas en México, que fluctúa entre 100 y 300 kilocalorias para distintos grupos de edad, según los análisis de las Encuestas de Nutrición. No nos queda clara la razón por la que a los responsables de la carta al editor les resulte extraño que los aumentos sean diferentes en México y en Estados Unidos.

Punto 9. Los responsables de la carta afirman que no seguimos la NOM-043SSA2-2205 al afirmar que existen alimentos que aumentan el riesgo de enfermedades crónicas. Consideramos que las Normas Oficiales Mexicanas (NOM) no deben ser consideradas como dogmas de fe y menos aún por la comunidad científica. Muchas de nuestras NOM requieren actualizaciones como resultado de las transiciones epidemiológica y nutricional y debido a la generación de nuevos conocimientos y nueva evidencia científica. Resulta muy claro, a partir de la evidencia generada en años recientes, que varios ingredientes de los alimentos tienen efectos adversos en la salud. Los ácidos grasos trans, los ácidos grasos saturados, el sodio, el azúcar, son todos ingredientes que cuando son aportados en exceso por algún alimento aumentan riesgos a la salud. Por tanto, es la responsabilidad de los científicos y los gobiernos proteger a los consumidores proporcionándoles información sobre la conveniencia de restringir el consumo de dichos alimentos. Esto debe hacerse aun si se afectan intereses económicos. Es importante que las NOM no se conviertan en motivo de censura y de control sobre afirmaciones relacionadas con los efectos de ingredientes de alimentos en la salud. Existe la necesidad de revisar periódicamente las NOM a la luz de nueva evidencia científica y nuevas realidades epidemiológicas.

Juan A Rivera, MS, PhD (I) jrivera@insp.mx

Onofre Muñoz-Hernández, $M$ en $C^{(2)}$ Martín Rosas-Peralta, MC, MCS, DCS(3) Carlos A Aguilar-Salinas, M Esp (4) Barry M Popkin, $P h D^{(5)}$ Walter CWillett, MD, PhD(6)

(I) Instituto Nacional de Salud Pública. Cuernavaca, Morelos, México

(2) Hospital Infantil de México Federico Gómez. México, DF

(3) Instituto Nacional de Cardiología Ignacio Chávez. México DF

(4) Instituto Nacional de Ciencias Médicas y Nutrición Salvador Zubirán. México DF

(5) School of Public Health, University of North Carolina. Chapel Hill, NC, EUA

(6) Harvard School of Public Health. Boston, MA, EUA

\section{Referencias}

I. Rivera JA, Muñoz-Hernández O, Rosas-Peralta M,Aguilar-Salinas CA, Popkin BM,Willett WC. Consumo de bebidas para una vida saludable: recomendaciones para la población mexicana. Salud Publica Mex 2008;50:173-195

2. Ludwig DS, Peterson KE. Relation between consumption of sugar sweetened drinks and childhood obesity: A prospective, observational analysis. Lancet 200 I;357(9255):505-508. 3. Raben A, Vasilaras TH. Sucrose compared with artificial sweeteners: Different effects on ad libitum food intake and body weight after $10 \mathrm{wk}$ of supplementation in overweight subjects. Am J Clin Nutr 2002;76(4):72I-729.

4. Schulze MB, Manson JE. Sugar-sweetened beverages, weight gain, and incidence of type 2 diabetes in young and middle-aged women. JAMA 2004:292(8):927-934

5. Malik VS, Schulze MB. Intake of sugar-sweetened beverages and weight gain:A systematic review. Am J Clin Nutr 2006;84(2):274-288.

6.Vartanian LR, Schwartz MB. Effects of soft drink consumption on nutrition and health:A systematic review and meta-analysis. Am J Public Health 2007;97(4):667-675.

7. Palmer JR, Boggs DA; Krishnan S, Hu FB, Singe $r$, Rosenberg, L Sugar-Sweetened Beverages and Incidence of Type 2 Diabetes Mellitus in African American Women. Arch Intern Med. 2008; 168(14): I 487- 1492.

8. Stevens G, Dias RH, Thomas KJA, Rivera JA, Carvalho N, et al. . Characterizing the epidemiological transition in Mexico: National and subnational burden of diseases, injuries, and risk factors. PLoS Med 2008; 5(6): el25. doi: I0.137I/ journal.pmed.0050I 25
9. Popkin BM,Armstrong LE, Bray GM, Caballero $B$, Frei B, Willett WC.A new proposed guidance system for beverage consumption in the United States. Am J Clin Nutr 2006;83:529-542.

I0. Frenk J.Tender puentes: lecciones globales desde México sobre políticas de salud basadas en evidencias. Salud Publica Mex 2007;49(I). I I. Pang T, Sudana R, Hanney S, Bhutta ZA, Hyder AA, Simon J. Knowledge for better health- $A$ conceptual framework and foundation for health research systems. Bull World Health Organ 2003; 8I: (II).

12. Haines A, Kuruvilla S, Burchert M. Bridging the implementation gap between knowledge and action for health. BullWorld Health Organ. 2004;82(I0). 13. World Cancer Fund/American Institute for Cancer Research. Food, Nutrition, Physical Activity, and the Prevention of Cancer: a Global Perspective.Washington DC:AICR, 2007. 14. DellaValle DM, . Roe LS et al. . Does the consumption of caloric and non-caloric beverages with a meal affect energy intake?" Appetite 2005;44(2):187-193.

15. Ledikwe JH, Blanck HM, et al. "Dietary energy density determined by eight calculation methods in a nationally representative United States population." J Nutr 2005; I 35(2):273.

16. Mattes RD. Dietary compensation by humans for supplemental energy provided as ethanol or carbohydrate in fluids. Physiol Behav 1996;59(I):I79-187.

17. Health D. G.A. C. R. N. a.Y. Dietary Guidelines for Americans. 6th ed.Washington DC:The Department of Health and Human Services [HHS] and the Department of Agriculture [USDA], 2005

18. Jones C,Woods K . Sugar, drinks, deprivation and dental caries in 14-year-old children in the north west of England in 1995. Community Dent Health 1999;16(2):68-7I.

19. Tam CS, Garnett SP. Soft drink consumption and excess weight gain in Australian school students: Results from the Nepean study. Int J Obes 2006;30(7): 1091-1093.

20. Sanigorski AM, Bell AC.Association of key foods and beverages with obesity in Australian schoolchildren. Public Health Nutr 2007; I0(2):I52-I57.

21. Bazzano LA, Tricia YL, Joshipura K J, Hu FB. Fruit and vegetable intake and risk of diabetes in women. Boston: Harvard University Department of Nutrition, 2007.

22.Villalpando S, Shamah T, Rivera J, Lara Y, Monterrubio E. Fortifying milk with ferrous gluconate and zinc oxide in a public nutrition program reduced the prevalence of anemia in toddlers. J Nutr 2006; I36:2633-2637.

23. Rivera JA, Sotrés-Alvarez D, Habicht JP, Shamah T,Villalpando S. Impact of the Mexican Program for Education, Health and Nutrition (Progresa) on rates of growth and anemia in infants and young children. A randomized effectiveness study. JAMA 2004;29I:2563-2570. 
24. Kaitosaari T, Ronnemaa T. Effect of 7-year infancy-onset dietary intervention on serum lipoproteins and lipoprotein subclasses in healthy children in the prospective, randomized Special Turku Coronary Risk Factor Intervention Project for Children (STRIP) study. Circulation 2003; 108(6):672-677.

25. Kaitosaari T, Ronnemaa T. Low-saturated fat dietary counseling starting in infancy improves insulin sensitivity in 9-year-old healthy children: The Special Turku Coronary Risk Factor Intervention Project for Children (STRIP) study. Diabetes Care 2006;29(4):78I-785.

26. Gidding SS. Dietary recommendations for children and adolescents:A guide for practitioners: Consensus statement from the American Heart Association. Circulation 2005; I I2(I3):206I-2075. (Endorsed by the American Academy of Pediatrics)

27.Victora CG, Adair L, Fall C, Hallal PC, Martorell R, Richter L, Sachdev H. Singh, for the Maternal and Child Undernutrition Study Group. Maternal and child undernutrition: consequences for adult health and human capital. Lancet 2008; Published Online January 17 DOI: 10.10161 SOI40-6736(07)61692-4.

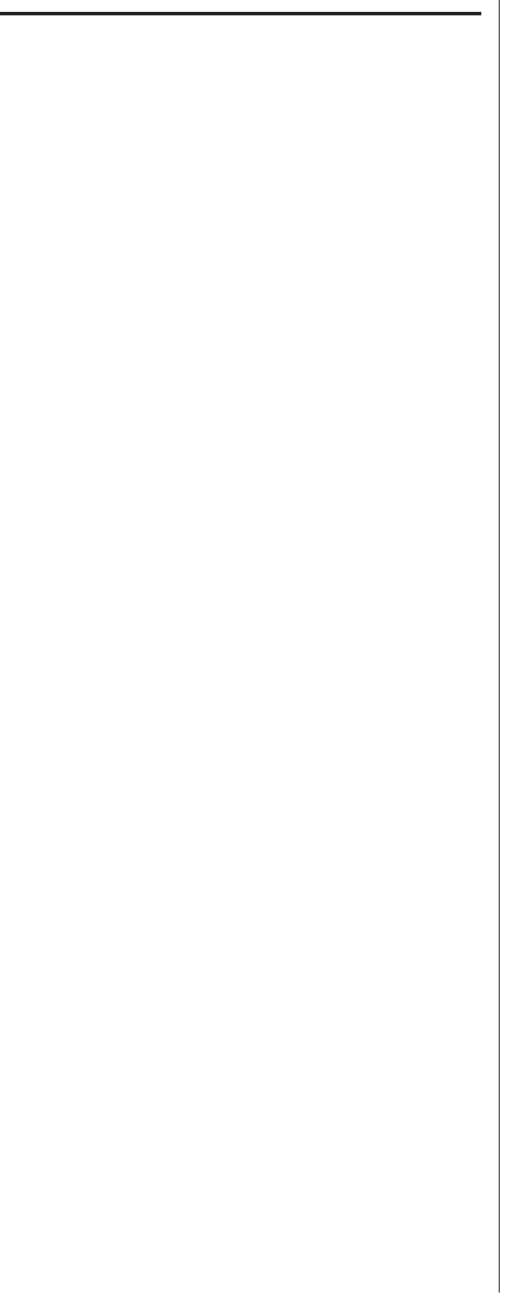

